\title{
Alternative configuration interaction expansions for transition metal ions with intermediate oxidation states in crystals: The structure and absorption spectrum of $\mathrm{Cs}_{2} \mathrm{GeF}_{6}: \mathrm{Mn}^{4+}$
}

\author{
Zoila Barandiarán ${ }^{\text {a) }}$ and Luis Seijo \\ Departamento de Química, C-XIV and Instituto Universitario de Ciencia de Materiales Nicolás Cabrera, \\ Universidad Autónoma de Madrid, 28049 Madrid, Spain
}

(Received 9 July 2001; accepted 27 July 2001)

\begin{abstract}
The vertical absorption spectrum of the $\mathrm{MnF}_{6}^{2-}$ cluster embedded in the $\mathrm{Cs}_{2} \mathrm{GeF}_{6}$ host crystal was recently calculated using the averaged coupled pair functional method leading to very large discrepancies with accurate one- and two-photon spectra. The same multiconfigurational expansions had previously been successful in similar systems which involved transition metal impurities in lower oxidation states. In this paper we show that the ligand-to-metal charge transfer configurations become so important in this intermediate oxidation state impurity (and, possibly, non-negligible ligand-ligand weak bonding interactions) that none of the 18 molecular orbitals of $\mathrm{F} 2 p$ character should be left inactive in the correlation treatment. This requirement can be satisfied in $\mathrm{MnF}_{6}^{2-}$ because of the higher oxidation state of manganese, which enhances the ligand field splittings in the Mn $3 d^{3}$ configuration manifold so much that one dominant Mn $3 d^{3}$ configuration rather than the full $\mathrm{Mn} 3 d^{3}$ active space can be used as a single reference for single and double excitations from all occupied ligand $2 p$ orbitals. The results of this work, together with those of previous studies, outline two different alternative truncation schemes of the valence electron correlation which produce the same, necessary, high accuracy in structural and spectroscopic properties of transition metal ions doped in ionic crystals. Whether one or the other should be used depends, basically, on the formal oxidation state of the transition metal impurity. (C) 2001 American Institute of Physics.
\end{abstract}

[DOI: $10.1063 / 1.1404140$ ]

\section{INTRODUCTION}

The study of the local distortions and spectroscopy of transition metal impurities in ionic crystals has been the subject of a series of research works in our laboratory since the formulation of the $a b$ initio model potential (AIMP) embedded cluster method. ${ }^{1}$ The solid state calculations are done using molecular methods and programs to calculate the electronic structure of the point defect cluster (e.g., $\mathrm{MnF}_{6}^{2-}$ in the $\mathrm{Cs}_{2} \mathrm{GeF}_{6}: \mathrm{Mn}^{4+}$ solid) being the quantum mechanical embedding host effects embodied in one-electron effective potentials which can be easily incorporated into the molecular cluster Hamiltonian. The good quality of the AIMP embedding has been repeatedly demonstrated over a series of applications $^{2}$ which showed that the capabilities and limitations of the solid state calculations are the same as those found in isolated molecules using standard quantum chemistry methods. These methods should be expected to be appropriate for local states of doped materials because, on the one hand, many impurities of basic and applied interest (transition metal, $f$ element ions) are open shell systems and, on the other hand, the properties which make them useful (as laser, scintillator, upconversion materials, etc.) are related to their excited state manifolds.

The study of the excited states of these materials is interesting and challenging for different reasons. Very high

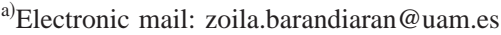

quality experimental data are often available, which offers a means to validate the methods. Some theoretical results can be complementary because they are not attainable or very difficult to obtain from experiment; good examples are the local structure of the point defects or the interpretation of some complex mechanisms of energy transfer between excited states which are very important to understand spectroscopic properties of technical interest such us those which favor or inhibit laser emissions. Moreover, an enormous variety of materials may result by combining different impurity ions with different hosts, which means an almost endless source of interesting properties and research.

However, the open shell nature and the high coordination number of the impurity [e.g., Mn(IV) in an octahedral site in $\left.\mathrm{Cs}_{2} \mathrm{GeF}_{6}: \mathrm{Mn}^{4+}\right]$ lead to difficulties because correlation of all valence electrons is often not feasible, so that choosing the truncation of the wave function expansions becomes very important. Along this line, a series of investigations conducted in our laboratory has pointed out that considerably different multiconfigurational expansions of the defect cluster wave functions are needed depending, basically, on the formal oxidation state of the impurity. ${ }^{3,4}$ This is related to the fact that the interactions between $d^{n}$ and ligand-to-metal charge transfer configurations increase dramatically with formal oxidation state: They are small in $\mathrm{V}(\mathrm{II}),{ }^{5,6}$ considerable in $\mathrm{Mn}(\mathrm{IV}),{ }^{4}$ and very large in $\mathrm{Fe}(\mathrm{VI})^{3}$ impurities, for instance. Accordingly, the truncations of the 
configurational space needed to properly account for electron correlation cannot be the same.

In particular, a recent calculation of the vertical absorption spectrum of the $\mathrm{MnF}_{6}^{2-}$ cluster embedded in the $\mathrm{Cs}_{2} \mathrm{GeF}_{6}$ host $^{4}$ using the averaged coupled pair functional $(\mathrm{ACPF})^{7}$ led to very large discrepancies with accurate oneand two-photon spectra, ${ }^{8-10}$ even though the same multireferences and truncated expansions had previously been used successfully in similar systems involving transition metal impurities in lower oxidation states. On the other hand, the $\mathrm{Mn}(\mathrm{IV})$ impurity could not be handled as in the high oxidation state limit [e.g., $\mathrm{Fe}(\mathrm{VI})$ in tetraoxo complexes in the solid state and aqueous solution $\left.{ }^{3}\right]$ where the quasidegeneracy effects are more important than dynamic correlation and long multiconfigurational self-consistent field (MCSCF) wave functions are indicated. Trying to under stand this failure, ACPF calculations were done using different multireferences and different subsets of active ligand orbitals in the correlation treatment, but their results showed very large variations of the transition energies: ${ }^{4} A_{2 g} \rightarrow{ }^{4} T_{2 g}: 21700-29450 \mathrm{~cm}^{-1} ; \quad{ }^{4} A_{2 g} \rightarrow a^{4} T_{1 g}: 30960-$ $38270 \mathrm{~cm}^{-1}$; compared to 22000 and $27300 \mathrm{~cm}^{-1}$ experimental values, respectively. The origins of the breakdown of the usual multireference treatment remained unrevealed and this, together with the instability of the results with the truncated space, was considered to be a significant methodological failure which needed to be communicated and further studied. $^{4}$

In effect, if the wave function based ab initio theoretical methods are meant to share with experimental techniques a significant role as tools for understanding the properties of these materials, it is necessary to find reasonable systematic configuration interaction truncation criteria which ultimately lead to uniform quality of both structural and spectroscopic properties irrespective of the oxidation state of the impurity. The work we present in this paper contributes in this line by proposing an alternative CI truncation for intermediate oxidation state $\mathrm{Mn}(\mathrm{IV})$ which leads to the same high accuracy for the structure and spectroscopy of $\mathrm{Cs}_{2} \mathrm{GeF}_{6}: \mathrm{Mn}^{4+}$ as that obtained so far for low oxidation state impurities doped in ionic hosts.

In summary, this and previous works show that two different alternative $\mathrm{CI}$ truncation procedures can be used which ultimately lead to comparable accuracy: In one procedure the complete $3 d^{n}$ active space is used as multireference for truncated CI expansions where correlation of only a subset of ligand $n p$ electrons is considered; this being applicable to low oxidation state impurities. In the other procedure, only the leading $3 d^{n}$ configuration is used as a single reference for CI expansions which include electron correlation of all ligand $n p$ electrons; this being applicable to intermediate oxidation state impurities like Mn(IV). The limit of high oxidation states seems to be essentially controlled by quasidegeneracy effects rather than by dynamic correlation. ${ }^{3}$

The details of the calculations and of the truncation procedures as well as their results are presented in Sec. II. The conclusions of this work are given in Sec. III.

\section{METHOD AND RESULTS}

\section{A. Basis sets and core/embedding AIMP}

In this paper we use the ab initio model potential method as an embedding technique and as an effective core potential method. ${ }^{2}$ The embedding potential we use for $\mathrm{Cs}_{2} \mathrm{GeF}_{6}$ was obtained in Ref. 4. The spin free relativistic Cowan-GriffinAIMP we use for Mn was obtained in Ref. 11 and represents its $[\mathrm{Mg}]$ core; the basis set for the $3 p 3 d 4 s$ valence being the $(9 s 5 p 5 d)$ primitive set, ${ }^{11}$ augmented by one diffuse $d$ function $^{12}$ and three $f$-type functions, ${ }^{13}$ contracted as $[4 s 3 p 5 d 1 f]$. The $[\mathrm{He}]$ core and primitive valence basis set for $\mathrm{F}$ were taken from Ref. 14; the $(5 s 5 p)$ set of primitive functions, augmented by one diffuse $p$ function for anions optimized for fluoride crystals in $\mathrm{KMgF}_{3}: \mathrm{F}^{-}$embedded ion calculations ${ }^{15}$ and $1 d$ polarization function, ${ }^{16}$ was contracted as $[2 s 3 p 1 d]$.

The whole set of embedding potentials, core potentials, and valence basis sets used in this work was carefully examined in Ref. 4 to detect any possible deficiencies. The high quality of the embedding was demonstrated by comparing the results of $\mathrm{Cs}_{2} \mathrm{GeF}_{6}: \mathrm{MnF}_{6}^{2-}$ calculations with those of a larger cluster: $\mathrm{Cs}_{2} \mathrm{GeF}_{6}: \mathrm{MnF}_{6} \mathrm{Cs}_{8}^{6+}$. And the results of the $\mathrm{Mn}$ and $\mathrm{F}$ core AIMPs and valence basis sets were compared to embedded $\mathrm{MnF}_{6}^{2-}$ calculations where all $81 \mathrm{Mn}$ and $\mathrm{F}$ electrons were treated explicitly and described by a much larger atomic natural orbital basis set: $\mathrm{Mn}(21 s 15 p 10 d 6 f 4 g),{ }^{17,18} \mathrm{~F}(14 s 9 p 4 d),{ }^{19}$ contracted as $\mathrm{Mn}[8 s 7 p 6 d 2 f 1 g], \mathrm{F}[4 s 3 p 2 d]$. In the end the test calculations indicated that the errors in the electronic transition energies associated with basis set limitations and use of core and embedding AIMP operators are smaller than $400 \mathrm{~cm}^{-1}$.

For access to AIMP data libraries which contain the basis sets and core/embedding potentials used here, see Ref. 20. The calculations have been done with the MOLCAS-5 package. ${ }^{21}$

\section{B. Multireference $\mathrm{Cl}$ expansions for low oxidation state impurities}

The multireference CI expansions which have been used successfully in low (I-III) oxidation state transition metal ion impurities in halide and oxide hosts ${ }^{2}$ and which breakdown in $\mathrm{Mn}(\mathrm{IV})$ are described here for the $\mathrm{Cs}_{2} \mathrm{GeF}_{6}: \mathrm{Mn}^{4+}$ material. The truncation criteria were proposed by Pierloot and Vanquickenborne in a detailed study of the correlation effects on the ligand field spectrum of the hexafluorochromate (III) anion. ${ }^{22}$

In a first step, complete active space self-consistent (CASSCF) field calculations ${ }^{23}$ are done which consider the molecular orbitals of main character Mn $3 d\left(t_{2 g}\right.$ and $e_{g}$ in the $O_{h}$ site symmetry) as the active space: $\operatorname{CASSCF}(3 d)$. Within this active space, only the lowest electronic states, which are associated with the so-called $10 D_{q}$ transition $\left(t_{2 g} \rightarrow e_{g}\right)$, are monoconfigurational: $t_{2 g}^{3}-{ }^{4} A_{2 g}$ (ground state) and $t_{2 g}^{2} e_{g}-{ }^{4} T_{2 g}$ (first spin quartet excited state); all others are mixtures of 2-5 $t_{2 g}^{x} e_{g}^{y}(x+y=3)$ configuration state functions. In a second step, the whole $\operatorname{CASSCF}(3 d)$ configurational space is taken as the reference for averaged coupled pair functional ${ }^{7}$ calculations aimed at the accurate 
TABLE I. CASSCF( $3 d$ ) results for $d^{3}$ impurities doped in fluoride hosts. Weights of the $t_{2 g}^{2} e_{g}$ and $t_{2 g} e_{g}^{2}$ open-shell configurations in the $\operatorname{CASSCF}(3 d)$ wave functions and transition energies from ${ }^{4} A_{2 g}$ (in cm ${ }^{-1}$ ) for the spin-quartet electronic states. In the ${ }^{4} T_{1 g}$ block the orbitals were optimized in the average energy of the two roots except when indicated.

\begin{tabular}{|c|c|c|c|c|c|}
\hline Impurity & Host & & ${ }^{4} T_{2 g}$ & $a^{4} T_{1 g}$ & $b^{4} T_{1 g}$ \\
\hline V(II) & $\mathrm{KMgF}_{3}$ & $\begin{array}{l}R(\mathrm{~V}-\mathrm{F})=2.074 \AA \\
t_{2 g}^{2} e_{g} \text { weight } \\
t_{2 g} e_{g}^{2} \text { weight } \\
\text { Transition energies }\end{array}$ & $\begin{array}{l}1.00 \\
10800\end{array}$ & $\begin{array}{l}0.61 \\
0.39 \\
17900\end{array}$ & $\begin{array}{l}0.39 \\
0.61 \\
29600\end{array}$ \\
\hline $\mathrm{Cr}(\mathrm{III})$ & $\mathrm{KMgF}_{3}$ & $\begin{array}{l}R(\mathrm{Cr}-\mathrm{F})=1.922 \AA \\
t_{2 g}^{2} e_{g} \text { weight } \\
t_{2 g} e_{g}^{2} \text { weight } \\
\text { Transition energies }\end{array}$ & $\begin{array}{l}1.00 \\
13900\end{array}$ & $\begin{array}{l}0.68 \\
0.32 \\
22500\end{array}$ & $\begin{array}{l}0.32 \\
0.68 \\
36300\end{array}$ \\
\hline $\mathrm{Mn}(\mathrm{IV})$ & $\mathrm{Cs}_{2} \mathrm{GeF}_{6}$ & $\begin{array}{l}R(\mathrm{Mn}-\mathrm{F})=1.77 \AA \\
t_{2 g}^{2} e_{g} \text { weight } \\
t_{2 g} e_{g}^{2} \text { weight } \\
\text { Transition energies } \\
t_{2 g}^{2} e_{g} \text { weight } \\
t_{2 g} e_{g}^{2} \text { weight } \\
\text { Transition energies }\end{array}$ & $\begin{array}{l}1.00 \\
24000\end{array}$ & $\begin{array}{l}0.88 \\
0.12 \\
34600 \\
0.91^{\mathrm{a}} \\
0.09^{\mathrm{a}} \\
33970^{\mathrm{a}}\end{array}$ & $\begin{array}{l}0.12 \\
0.88 \\
54200\end{array}$ \\
\hline
\end{tabular}

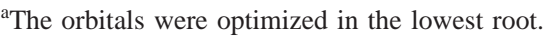

computation of the electronic absorption and emission transition energies. Since the number of valence electrons is very large and most of the $\operatorname{CASSCF}(3 d)$ wave functions are multiconfigurational, it is not possible, in general, to correlate all metal $3 d$ and ligand $2 p$ electrons but only the electrons occupying a subset of ligand orbitals. Pierloot and Vanquickenborne ${ }^{22}$ demonstrated that very good results compared to experiment can be obtained by correlating the $3 d$ electrons and the electrons which occupy the ligand orbitals transforming according to the same irreducible representations as the metal $3 d$, namely, the $t_{2 g}$ and $e_{g}$ molecular orbitals of ligand $2 p$ character. The application of this criterium to the $\mathrm{MnF}_{6}^{2-}$ cluster means that $\mathrm{ACPF}$ calculations are done correlating 13 electrons which occupy 10 active orbitals: the $t_{2 g}$ and $e_{g}$ molecular orbitals of F $2 p$ character and the $t_{2 g}$ and $e_{g}$ orbitals of Mn $3 d$ character.

Previous works on isoelectronic $\mathrm{V}^{2+}$-doped fluoroperovskites ${ }^{5,6,15}$ and $\mathrm{Cr}^{3+}$-doped elpasolites ${ }^{24,25}$ and fluoroperovskites ${ }^{26}$ [among other $d^{n}$ impurities: $d^{2} \mathrm{~V}(\mathrm{III}) ;{ }^{27}$ $\left.d^{4} \mathrm{Cr}(\mathrm{II}) ;{ }^{28,29} d^{8} \mathrm{Ni}(\mathrm{II}) ;{ }^{30} d^{9} \mathrm{Cu}(\mathrm{II}),{ }^{31} \mathrm{Me}(\mathrm{II}), \mathrm{Me}=\mathrm{Sc}-\mathrm{Zn}^{32}\right]$ have shown that the CASSCF( $3 d)$ level of calculation is very good for geometry optimizations of the ground and excited states. The agreement of the calculated electronic transitions at the ACPF-13 level with available experimental measurements of ground and excited state absorption, and emission spectra was found to be very high. When applied to $\mathrm{Cs}_{2} \mathrm{GeF}_{6}: \mathrm{Mn}^{4+}$, however, neither the geometry parameters nor the absorption spectrum showed the same systematic low error bounds when compared to very accurate experimental results. ${ }^{4,10}$

The totally symmetric vibrational frequency was found to be $19 \%$ too high compared to experiment: $704^{4}$ versus $592^{10} \mathrm{~cm}^{-1}$, respectively, in contrast to the typical $3 \%$ error bound of CASSCF $(3 d)$ predictions in other low oxidation state impurities: $\mathrm{KMgF}_{3}: \mathrm{V}^{2+}\left(545^{15}\right.$ versus $\left.550^{33} \mathrm{~cm}^{-1}\right)$ $\mathrm{KMgF}_{3}: \mathrm{Cr}^{3+}\left(576^{26}\right.$ versus $\left.562^{34} \mathrm{~cm}^{-1}\right), \mathrm{K}_{2} \mathrm{NaGaF}_{6}: \mathrm{Cr}^{3+}$ $\left(582^{24}\right.$ versus $\left.568^{35} \mathrm{~cm}^{-1}\right)$ among others. ${ }^{2}$ Also, the band maxima of the two spin-allowed bands observed at $22000 \mathrm{~cm}^{-1} \quad\left({ }^{4} A_{2 g} \rightarrow{ }^{4} T_{2 g}\right) \quad$ and $\quad 27300 \mathrm{~cm}^{-1} \quad\left({ }^{4} A_{2 g}\right.$ $\left.\rightarrow a^{4} T_{1 g}\right)^{10}$ were obtained at the ACPF-13 level too high by $3800(17 \%)$ and $7000 \mathrm{~cm}^{-1}(26 \%)$, respectively, whereas equivalent calculations lead to the following errors in isoelectronic impurities: below $3 \%$ for the $10 D_{q}$ transition ${ }^{4} A_{2 g} \rightarrow{ }^{4} T_{2 g}$ and below $8 \%$ for the next higher excitation ${ }^{4} A_{2 g} \rightarrow a{ }^{4} T_{1 g} \cdot{ }^{15,24,26}$

The origins of the breakdown in $\mathrm{Cs}_{2} \mathrm{GeF}_{6}: \mathrm{Mn}^{4+}$ were investigated by performing a variety of complete and restricted active space self-consistent field (CASSCF, RASSCF) ${ }^{23}$ calculations which, on the one hand, could reveal the actual importance of ligand-to-metal charge transfer configurations, and, on the other hand, could help to decide upon new multireference CI schemes. The results of those CAS/RASSCF calculations pointed out the importance of the interactions between $d^{n}$ and ligand-to-metal charge transfer configurations, which were found to be considerable, state specific, to increase from the ground to the higher excited state, and to occur mostly through the F $2 p t_{2 g} \rightarrow \mathrm{Mn} 3 d t_{2 g}$ and F $2 p \quad e_{g} \rightarrow \mathrm{Mn} 3 d e_{g}$ excitations. Accordingly, a set of ACPF calculations were done based either on the $\operatorname{CASSCF}(3 d)$ multireference or on larger references including the most important charge transfer configurations as well. In all these calculations, though, the number of ligand $2 p$ electrons which were correlated was limited and never greater than 10 (those electrons occupying the F $2 p t_{2 g}$ and $e_{g}$ molecular orbitals). All these calculations that use large multireferences failed to give stable and uniformly good results for the two test case transitions. ${ }^{4}$

Another possible source of error was mentioned in Ref. 4 related to the observed trend of the impurity-ligand bond lengths calculated in the isoelectronic series: $\mathrm{KMgF}_{3}: \mathrm{V}^{2+}$ $(2.074 \AA), \mathrm{KMgF}_{3}: \mathrm{Cr}^{3+}(1.922 \AA), \mathrm{Cs}_{2} \mathrm{GeF}_{6}: \mathrm{Mn}^{4+}(1.76$ $\AA$ ). The F-F distances decrease accordingly from 2.933 to 
TABLE II. Equilibrium distance, $R_{e}$, and totally symmetric vibrational frequency, $\bar{\nu}_{a_{1 g}}$, of the ${ }^{4} A_{2 g}$ ground state of $\mathrm{Cs}_{2} \mathrm{GeF}_{6}: \mathrm{Mn}^{4+}$.

\begin{tabular}{llll}
\hline \hline & CASSCF $(3 d)$ & \multicolumn{1}{c}{ CPF-39 } & Experiment $^{\mathrm{a}}$ \\
\hline$R_{e} / \AA$ & 1.761 & 1.788 & $\ldots$ \\
$\bar{\nu}_{a_{1 g}} / \mathrm{cm}^{-1}$ & $704(19 \%)$ & $607(2.5 \%)$ & 592 \\
\hline \hline
\end{tabular}

${ }^{\mathrm{a}}$ Reference 10.

2.718 and $2.49 \AA$ in the series. According to Pauling ${ }^{36}$ the van der Waals radius of $\mathrm{F}$ is $1.35 \AA$. Thus, it was pointed out that the usual truncation scheme might be failing to describe possible non-negligible $\mathrm{F}-\mathrm{F}$ weak bonding interactions in the tight $\mathrm{MnF}_{6}^{2-}$ cluster due to the truncation of the $\mathrm{F} 2 p$ electron correlation treatment.

\section{Alternative $\mathrm{Cl}$ expansions for intermediate oxidation state $\mathrm{Cs}_{2} \mathrm{GeF}_{6}: \mathrm{MnF}_{6}^{2-}$}

In this work, we decided to completely change the strategy of the CASSCF+ACPF calculations orienting the truncations in the opposite direction to give more relevance to the ligand excitations. The idea is that if ligand-to-metal charge transfer and ligand-ligand interactions are becoming important in $\mathrm{Cs}_{2} \mathrm{GeF}_{6}: \mathrm{Mn}^{4+}$, all electrons occupying molecular orbitals of main ligand $2 p$ character should be correlated at the ACPF level, even if this is achieved at the expense of truncating the multireference.

As can be seen in Table I, the alternative of truncating the $\operatorname{CASSCF}(3 d)$ multireference down to one configuration becomes plausible just as the oxidation state grows from $\mathrm{V}$ (II) to $\mathrm{Mn}(\mathrm{IV})$ : The ligand field splitting of the $3 d$ orbitals becomes larger (cf. the electronic transitions) and the CAS $(3 d)$ manifold increasingly decoupled, as illustrated for the $2 \times 2{ }^{4} T_{1 g}$ block. The dominance of one of the $d^{3}$ configurations in other electronic states of $\mathrm{Cs}_{2} \mathrm{GeF}_{6}: \mathrm{Mn}^{4+}$ is illustrated by its weight in the $\operatorname{CASSCF}(3 d)$ wave function, which is included in Table III (see the following).

In Tables II and III we present the results of the structure and ground state absorption spectrum of $\mathrm{Cs}_{2} \mathrm{GeF}_{6}: \mathrm{Mn}^{4+}$ as calculated by correlating all the $36 \mathrm{~F} 2 p$ and $3 \mathrm{Mn} 3 d$ valence electrons; we will refer to these (single reference) coupled pair functional ${ }^{7}$ calculations as CPF-39. The single reference configuration and the orbitals used in the CPF-39 are obtained in preparatory $\operatorname{CASSCF}(3 d)$ calculations where the orbitals are optimized in the lowest root. The weight of

TABLE III. Vertical electronic transitions of $\mathrm{Cs}_{2} \mathrm{GeF}_{6}: \mathrm{Mn}^{4+}$ calculated at the $\mathrm{Mn}-\mathrm{F}$ equilibrium distance of the ${ }^{4} A_{2 g}$ ground state $1.788 \AA$. All numbers are in $\mathrm{cm}^{-1}$.

\begin{tabular}{llllll}
\hline \hline & \multicolumn{2}{c}{ Reference (weight) } & CPF-39 & $+3 p$ pol $^{\mathrm{a}}$ & Experiment $^{\mathrm{b}}$ \\
\hline${ }^{4} A_{2 g}$ & $t_{2 g}^{3}$ & $(1.00)$ & 0.0 & 0.0 & 0.0 \\
${ }^{4} T_{2 g}$ & $t_{2 g}^{2} e_{g}$ & $(1.00)$ & 23500 & 22720 & 22000 \\
$a^{4} T_{1 g}$ & $t_{2 g}^{2} g_{g}$ & $(0.91)$ & 29340 & 26500 & 27300 \\
${ }^{2} E_{g}$ & $t_{2 g}^{3}$ & $(0.97)$ & 17490 & 15670 & 16028 \\
${ }^{2} T_{1 g}$ & $t_{2 g}^{3}$ & $(0.98)$ & 17920 & 16540 & 16780,16835 \\
\hline
\end{tabular}

${ }^{a}$ The CPF-39 configurational space is extended so as to include all single excitations from the molecular orbitals of $\mathrm{Mn} 3 p$ character.

${ }^{\mathrm{b}}$ References 8-10. the (leading) reference configuration in the $\operatorname{CASSCF}(3 d)$ wave functions is tabulated for each electronic state in parentheses in Table III.

The quality of the structural results can be assessed by comparing the totally symmetric stretching mode $\bar{\nu}_{a_{1 g}}$ with experiment. The usual accuracy in the $\bar{\nu}_{a_{1 g}}$ is now reached, the error of the CPF-39 result being $2.5 \%$. Analogously, the large discrepancies obtained with the previous CASSCF $+\mathrm{ACPF}(13)$ procedure of $3800 \mathrm{~cm}^{-1}\left({ }^{4} A_{2 g} \rightarrow{ }^{4} T_{2 g}\right)$ and $7000 \mathrm{~cm}^{-1}\left({ }^{4} A_{2 g} \rightarrow a^{4} T_{1 g}\right)$ now turn into acceptable 1500 and $2000 \mathrm{~cm}^{-1}$ deviations from the experimental band maxima when calculated at the CPF-39 level at the CPF-39 equilibrium Mn-F distance $1.788 \AA$ (see Table III). Similar errors are obtained for the spin-forbidden transitions, which sets the overall error bound to $2000 \mathrm{~cm}^{-1}$. In addition, when the CPF-39 configurational space is enlarged so as to include single excitations from the molecular orbitals of main character Mn $3 p$, which allows these orbitals to relax (results labeled " $+3 p$ pol" in Table III), the discrepancies become even smaller. These results coincide, within $500 \mathrm{~cm}^{-1}$, with CPF-45 calculations where single and double excitations from the molecular orbitals of Mn $3 p$ character were allowed. It is known that outer core $3 p$ correlation may selectively affect the ligand field transitions; $;^{2,37}$ but the similarity of the CPF-39+3p polarization to the CPF- 45 calculated electronic transitions found in this system indicates that it is only the $3 p$ core polarization that is primarily responsible for the final refinement of the absorption spectrum.

In order to reinforce the good behavior of the procedure proposed by Pierloot and Vanquickenborne ${ }^{22}$ in low oxidation state impurities, we have recalculated in $\mathrm{KMgF}_{3}: \mathrm{V}^{2+}$ and $\mathrm{KMgF}_{3}: \mathrm{Cr}^{3+}$ the only truly single-reference electronic transition, ${ }^{4} A_{2 g} \rightarrow{ }^{4} T_{2 g}$, at the CPF-39 level. The results $\left(11330 \mathrm{~cm}^{-1}\right.$ for $\mathrm{KMgF}_{3}: \mathrm{V}^{2+}$ and $15110 \mathrm{~cm}^{-1}$ for $\mathrm{KMgF}_{3}: \mathrm{Cr}^{3+}$ ) deviate from the previous calculations by less than $250 \mathrm{~cm}^{-1}, 5,26$ as expected from their low oxidation states.

\section{CONCLUSIONS}

In this paper we have calculated the ground state structural parameters and the absorption spectrum of the $\mathrm{Cs}_{2} \mathrm{GeF}_{6}: \mathrm{Mn}^{4+}$ crystal using the AIMP embedded cluster method. It has been shown that, associated with its higher formal oxidation state, the $3 d^{3}$ configuration manifold is very effectively decoupled compared to isoelectronic impurities of lower oxidation state. This circumstance makes it possible to correlate all $\mathrm{F} 2 p$ and Mn $3 d$ electrons through size consistent single and double excitations from only one (leading) $d^{3}$ configuration. The results obtained are in very good agreement with one- and two-photon absorption spectra, in contrast to previous multireference truncated CI expansions, where selective treatment of the $\mathrm{F} 2 p$ electrons was imposed, which led to very large discrepancies with experiments. This is interpreted as an indication that the effects of ligand-to-metal charge transfer configurations and of ligand-ligand weak bonding interactions are described in a balanced way in the new truncation procedure proposed in this paper as an alternative for the accurate study of interme- 
diate oxidation state transition metal ions like $\mathrm{Mn}(\mathrm{IV})$. Alternative procedures to truncate $\mathrm{CI}$ and MCSCF expansions have been proposed elsewhere which are known to be adequate for low and for very high oxidation state transition metal impurities, but both of them completely break down in the $\mathrm{Cs}_{2} \mathrm{GeF}_{6}: \mathrm{Mn}^{4+}$ material. Therefore, the new scheme proposed in this paper serves to cover the gap set by the $\mathrm{Mn}(\mathrm{IV})$ impurity ion.

\section{ACKNOWLEDGMENT}

This work was partly supported by a grant from Ministerio de Ciencia y Tecnología, Spain (Dirección General de Investigación, PB98-0108).

${ }^{1}$ Z. Barandiarán and L. Seijo, J. Chem. Phys. 89, 5739 (1988).

${ }^{2}$ L. Seijo and Z. Barandiarán, in Computational Chemistry: Reviews of Current Trends, edited by J. Leszczynski (World Scientific, Singapore, 1999), Vol. 4, p. 55.

${ }^{3}$ A. Al-Abdalla, L. Seijo, and Z. Barandiarán, J. Chem. Phys. 109, 6396 (1998).

${ }^{4}$ L. Seijo, Z. Barandiarán, and D. S. McClure, Int. J. Quantum Chem. 80, 623 (2000).

${ }^{5}$ S. López-Moraza and Z. Barandiarán, J. Chem. Phys. 105, 50 (1996).

${ }^{6}$ S. López-Moraza, L. Seijo, and Z. Barandiarán, Phys. Rev. B 57, 11974 (1998).

${ }^{7}$ R. Ahlrichs, P. Scharf, and C. Ehrhardt, J. Chem. Phys. 82, 890 (1985); R. J. Gdanitz and R. Ahlrichs, Chem. Phys. Lett. 143, 413 (1988).

${ }^{8}$ R-L. Chien, J. M. Berg, D. S. McClure, P. Rabinowitz, and B. N. Perry, J. Chem. Phys. 84, 4168 (1986).

${ }^{9}$ C. Campochiaro, D. S. McClure, P. Rabinowitz, and S. Dougal, Chem. Phys. Lett. 157, 78 (1989).

${ }^{10}$ C. Campochiaro, Ph.D. thesis, Princeton University, 1991.

${ }^{11}$ Z. Barandiarán and L. Seijo, Can. J. Chem. 70, 409 (1992).

${ }^{12}$ P. J. Hay, J. Chem. Phys. 66, 4377 (1977).

${ }^{13}$ L. G. M. Pettersson (private communication).

${ }^{14}$ S. Huzinaga, L. Seijo, Z. Barandiarán, and M. Klobukowski, J. Chem. Phys. 86, 2132 (1987).

${ }^{15}$ S. López-Moraza, J. L. Pascual, and Z. Barandiarán, J. Chem. Phys. 103, 2117 (1995).
${ }^{16}$ Gaussian Basis Sets for Molecular Calculations, edited by S. Huzinaga (Elsevier, Amsterdam, 1984).

${ }^{17}$ H. Partridge, J. Chem. Phys. 90, 1043 (1989).

${ }^{18}$ R. Pou-Amerigo, M. Merchán, P.-O. Widmark, and B. O. Roos (unpublished).

${ }^{19}$ P.-O. Widmark, P.-A. Malmqvist, and B. O. Roos, Theor. Chim. Acta 77, 291 (1990).

${ }^{20}$ Detailed core and embedding AIMP data libraries in electronic format are available from the authors upon request or directly at the address http://www.qui.uam.es/Data/AIMPLibs.html. See also Ref. 21.

${ }^{21}$ MOLCAS 5, K. Andersson, M. Barysz, A. Bernhardsson et al., Lund University, Sweden, (2000)

${ }^{22}$ K. Pierloot and L. G. Vanquickenborne, J. Chem. Phys. 93, 4154 (1990).

${ }^{23}$ B. O. Roos, P. R. Taylor, and P. E. M. Siegbahn, Chem. Phys. 48, 157 (1980); P. E. M. Siegbahn, A. Heiberg, J. Almlöf, and B. O. Roos, J. Chem. Phys. 74, 2384 (1981); P. Siegbahn, A. Heiberg, B. Roos, and B. Levy, Phys. Scr. 21, 323 (1980).

${ }^{24}$ L. Seijo, Z. Barandiarán, and L. G. M. Pettersson, J. Chem. Phys. 98, 4041 (1993).

${ }^{25}$ A. Al-Abdalla, Z. Barandiarán, L. Seijo, and R. Lindh, J. Chem. Phys. 108, 2005 (1998).

${ }^{26}$ S. López-Moraza, L. Seijo, and Z. Barandiarán, Int. J. Quantum Chem. 77, 961 (2000).

${ }^{27}$ A. Al-Abdalla, L. Seijo, and Z. Barandiarán, J. Mol. Struct.: THEOCHEM 451, 135 (1998)

${ }^{28}$ J. L. Pascual and L. Seijo, J. Chem. Phys. 102, 5368 (1995).

${ }^{29}$ J. L. Pascual, L. Seijo, and Z. Barandiarán, J. Chem. Phys. 103, 4841 (1995).

${ }^{30}$ R. Llusar, M. Casarrubios, Z. Barandiarán, and L. Seijo, J. Chem. Phys. 105, 5321 (1996).

${ }^{31}$ J. L. Pascual, L. Seijo, and Z. Barandiarán, J. Chem. Phys. 98, 9715 (1993).

${ }^{32}$ L. Seijo and Z. Barandiarán, Int. J. Quantum Chem. 60, 617 (1996).

${ }^{33}$ R. Moncorgé and T. Benyattou, Phys. Rev. B 37, 9177 (1988).

${ }^{34}$ M. Mortier, Q. Wang, J. Y. Buzaré, and M. Rousseau, Phys. Rev. B 56, 3022 (1997).

${ }^{35}$ J. Ferguson, H. J. Guggenheim, and D. L. Wood, J. Chem. Phys. 54, 504 (1971).

${ }^{36} \mathrm{~L}$. Pauling, The Nature of the Chemical Bond and the Structure of Molecules and Crystals: An Introduction of Modern Structural Chemistry (Cornell University Press, New York, 1960).

${ }^{37}$ K. Pierloot, E. Tsokos, and B. O. Roos, Chem. Phys. Lett. 214, 583 (1993). 